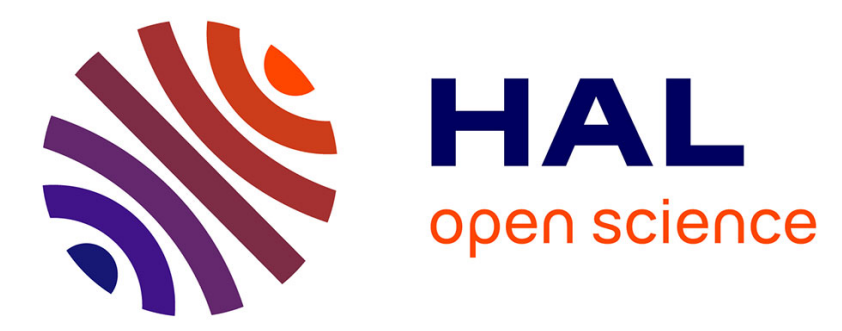

\title{
Particle Filter-Based Prognostics for an Electrolytic Capacitor Working in Variable Operating Conditions
}

\author{
Marco Rigamonti, Piero Baraldi, Enrico Zio, Daniel Astigarraga, Ainhoa
}

Galarza

\section{- To cite this version:}

Marco Rigamonti, Piero Baraldi, Enrico Zio, Daniel Astigarraga, Ainhoa Galarza. Particle FilterBased Prognostics for an Electrolytic Capacitor Working in Variable Operating Conditions. IEEE Transactions on Power Electronics, 2016, 31 (2), pp.1567 - 1575. 10.1109/TPEL.2015.2418198 . hal01265907

\section{HAL Id: hal-01265907 \\ https://hal.science/hal-01265907}

Submitted on 1 Feb 2016

HAL is a multi-disciplinary open access archive for the deposit and dissemination of scientific research documents, whether they are published or not. The documents may come from teaching and research institutions in France or abroad, or from public or private research centers.
L'archive ouverte pluridisciplinaire HAL, est destinée au dépôt et à la diffusion de documents scientifiques de niveau recherche, publiés ou non, émanant des établissements d'enseignement et de recherche français ou étrangers, des laboratoires publics ou privés. 


\title{
Particle Filter-Based Prognostics for an Electrolytic Capacitor Working in Variable Operating Conditions
}

\author{
Marco Rigamonti ${ }^{1}$, Piero Baraldi ${ }^{1}$, Enrico Zio $^{1,2}$, Daniel Astigarraga ${ }^{3}$, and Ainhoa Galarza ${ }^{3}$ \\ ${ }^{1}$ Dipartimento di Energia, Politecnico di Milano, Via Ponzio 34/3, Milan, 20133, Italy \\ marcomichael.rigamonti@polimi.it \\ piero.baraldi@polimi.it \\ enrico.zio@polimi.it \\ ${ }^{2}$ Chair on Systems Science and the Energetic Challenge, Ecole Centrale Paris and Supelec, France \\ enrico.zio@ecp.fr \\ ${ }^{3}$ CEIT, Manuel de Lardizabal 15, San Sebastian, 20018, Spain \\ dastigarraga@ceit.es \\ agalarza@ceit.es
}

Corresponding Author: Professor Enrico Zio;

Email address: enrico.zio@polimi.it

Postal address: Dipartimento di Energia - Politecnico di Milano - Via Lambruschini 4, 20156 Milano

Phone number: +39 0223996340

This paper has never been presented at a conference or submitted elsewhere previously.

\begin{abstract}
Prognostic models should properly take into account the effects of operating conditions on the degradation process and on the signal measurements used for monitoring. In this work, we develop a Particle Filter-based (PF) prognostic model for the estimation of the Remaining Useful Life (RUL) of aluminum electrolytic capacitors used in electrical automotive drives, whose operation is characterized by continuously varying conditions. The capacitor degradation process, which remarkably depends from the temperature experienced by the component, is typically monitored by observing the capacitor Equivalent Series Resistance (ESR). However, the ESR measurement is influenced by the temperature at which the measurement is performed, which changes depending on the operating conditions. To address this problem, we introduce a novel degradation indicator independent from the measurement temperature. Such indicator can, then, be used for the prediction of the capacitor degradation and its RUL. For this, we develop a Particle Filter prognostic model, whose performance is verified on data collected in simulated and experimental degradation tests.
\end{abstract}

Keywords: Fault diagnosis, Electrolytic capacitors, Monte Carlo methods, Bayes procedures, Reliability modeling 


\section{INTRODUCTION}

Prognostics of failures in engineered equipment is based on the capability of predicting future degradation paths, so as to estimate the Remaining Useful Life (RUL) of the equipment and the potential risks associated to its failure ([1], [2] and [3]). On this basis, it is possible to define predictive maintenance strategies to set the best maintenance actions for allowing the optimal exploitation of the useful life of the monitored equipment, with benefits in terms of reduction of costs and improvement of safety.

In practice, industrial equipment works in varying operating conditions. These variations can have remarkable effects on the degradation process and on the values of the signals measured to monitor it [4]. For example, structures operating in environments characterized by high temperatures usually show faster degradation than structures operating at low temperatures, whereas high temperatures may modify the measurement of electrical signals used to estimate the degradation of an electrical device. Thus, it is fundamental that prognostic methods properly take into account the effects of variations of operating conditions [5].

In this work, we consider model-based prognostic approaches, which use mathematical representations of the equipment degradation process to predict the equipment RUL. In model-based prognostics, it is possible to distinguish between two different situations: i) the effects of operating conditions on the degradation process and on the measured signals are known and represented in the mathematical models, ii) the effects are not fully known and a mathematical model of the operating conditions influence is not available. In the former situation i), traditional model-based prognostic approaches, such as those based on Bayesian Filters [6], can be directly used, whereas in the latter ii) properly tailored prognostic approaches are needed.

In the present work, we consider the second situation, with specific reference to the problem of predicting the RUL of aluminum electrolytic capacitors installed in Fully Electric Vehicles (FEVs) [5]. The main task of this component, which is the most commonly used electrolytic capacitors in the electronics industry [7], is to filter the voltage provided to the inverter of the electric motor [8]. According to [9], electrolytic capacitors are very critical components, being responsible for almost $30 \%$ of the total number of failures in electrical systems and, thus, it is 
of paramount importance to develop predictive maintenance approaches for them. The failure mechanisms of the aluminum electrolytic capacitors can be catastrophic or gradual. In case of catastrophic failures, the capacitor completely and abruptly loses its function due to short or open circuits, whereas gradual failures are characterized by a gradual functionality loss ([10], [11] and [12]). The main cause of this latter degradation mechanism, which is the most common in electrolytic capacitor, is the vaporization of electrolyte. This degradation process is strongly influenced by the capacitor operating conditions, such as voltage, current, frequency, and working temperature [13]. In capacitors installed in FEVs, these conditions tend to continuously change due to external factors such as season, geographical area, driving style. In particular, the temperature experienced by the capacitor, which depends on the applied loads and on the external temperature, has a remarkable influence on the evolution of the degradation process: higher the temperature, faster the vaporization process due to the increase of the self-heating effects [14].

Aluminum electrolytic capacitor degradation has been investigated by several authors. A direct degradation indicator for capacitors operating at constant temperature and load is the Equivalent Series Resistance (ESR) ([11], [15], [16] and [17]). A capacitor is considered failed, i.e. not able to properly accomplish to its functions, when its ESR exceeds the double of its initial value [18]. In [15], the main degradation mechanisms of a capacitor were analyzed and its equivalent circuit model developed. In [11], a degradation model based on the physics of the wear-out mechanism was presented. In [19], a method based on the use of genetic algorithm for the identification of the parameters of the degradation model was discussed. In [13], a method for studying the degradation effects of electrolytic capacitors subjected to loading under extreme operating conditions was proposed. Furthermore, some approaches for monitoring capacitor degradation and for predicting its RUL were proposed. In [20], a method for real-time monitoring and RUL prediction for electrolytic capacitor used in uninterruptible power supplies (UPSs) was developed.

However, the temperature at which the ESR measurement is performed has a remarkable influence on the ESR measurement (higher the temperature, lower the ESR), whereas the above models consider capacitors aging at constant temperature and do not quantify the uncertainty on their predictions. A Bayesian approach for the 
prediction of the capacitor RUL probability distribution has been proposed in [8], where a prognostic methodology based on the application of a Kalman Filter (KF) for tracking the capacitor health state, forecasting the capacitance evolution and predicting the capacitor RUL was presented. This approach does not consider the possibility of variable operating and environmental conditions and, as underlined by the authors themselves, it is not able to cope with the abrupt change of the capacitor functional behavior arising near the end of the component life, thus providing inaccurate RUL predictions [8].

The objective of the present work is to provide a method for the prediction of the RUL for a capacitor working in variable operating conditions. The method should also be able to estimate the uncertainty affecting the RUL prediction.

The two main novelties of the proposed prognostic method are:

1) the definition of a novel degradation indicator for capacitors operating at variable temperatures;

2) the implementation and application to electrolytic capacitors of a particle filtering approach for RUL uncertainty estimation.

The proposed degradation indicator is the ratio between the ESR measured on the degraded capacitor and the ESR value expected on a new capacitor at the same operational temperature. This index provides an indication of the capacitor degradation level and, since it is independent from the measurement temperature, it can be used for capacitors working in variable operating conditions. Its definition has required performing a series of laboratory experiments for investigating the relationship between ESR and temperature in a new capacitor.

The physics-based model of the ESR evolution proposed in [20] has been applied to the new degradation indicator and used within a sequential Bayesian approach for the estimation of component degradation. The Bayesian approach has been employed to account for the uncertainty affecting: i) the ESR and temperature measurement processes, ii) the possible inaccuracy of the degradation model, iii) the stochasticity of the degradation process. Since a classical Kalman Filter approach cannot be applied to this problem due to the presence of non-additive noise terms, we resort to a PF approach [21]. Once the component degradation state probability distribution has 
been estimated by the PF method, Monte Carlo (MC) simulation has been used for the prediction of the future component degradation path and its RUL [22]. The MC simulation allows to properly take into account the uncertainty on the present degradation state estimation and the uncertainty on the future evolution of the operating conditions.

The performance of the proposed prognostic method has been verified with respect to 1) numerical simulations of the capacitor degradation process and 2) degradation data collected in laboratory accelerated life tests.

The remaining part of the paper is organized as follows: in Section 2, the particle filtering approach for the RUL estimation is presented; Section 3 describes the proposed capacitor degradation model; in Section 4, the experimental test setup for the parameters estimation and the application of the developed method to both simulated and experimental data is discussed; finally, in Section 5 some conclusions and remarks are drawn.

\section{PARTICLE FILTER-BASED PROGNOSTICS}

We consider a situation in which a physics-based model of the degradation process is available and can be formulated in the form of a first-order Markov Process:

$$
x_{t}=g\left(x_{t-1}, \gamma_{t-1}\right)
$$

where $g(x, \gamma)$ is a recursive, possibly non-linear, transition function, $x_{t}$ is the indicator of the equipment degradation state at time $t$ and $\gamma_{t}$ is the process noise used to capture the degradation process stochasticity and the inaccuracy of the model.

We assume that the observation equation providing a link between the degradation state $x$ and its measurement $z$ is known and can be represented by a possibly non-linear function $h$ :

$$
z_{t}=h\left(x_{t}, \sigma_{t}\right)
$$

where $\sigma$ represents the measurement error. 
The PF-based approach to prognostics relies on the following three steps (Figure 1):

1. a filtering step for the estimation of the equipment degradation state at the present time, which is based on the use of Eqs. (1) and (2) and the measurements, $z_{1: t}$, performed until the present time

2. a prediction step for the estimation of the future degradation evolution using the posterior probability density function (pdf) of the degradation state (output of step 1) and the degradation model (Eq. 1))

3. the prediction of the equipment RUL considering the future degradation state prediction (output of step 2) and the failure threshold, i.e., the value of the degradation indicator above which the equipment is considered failed.

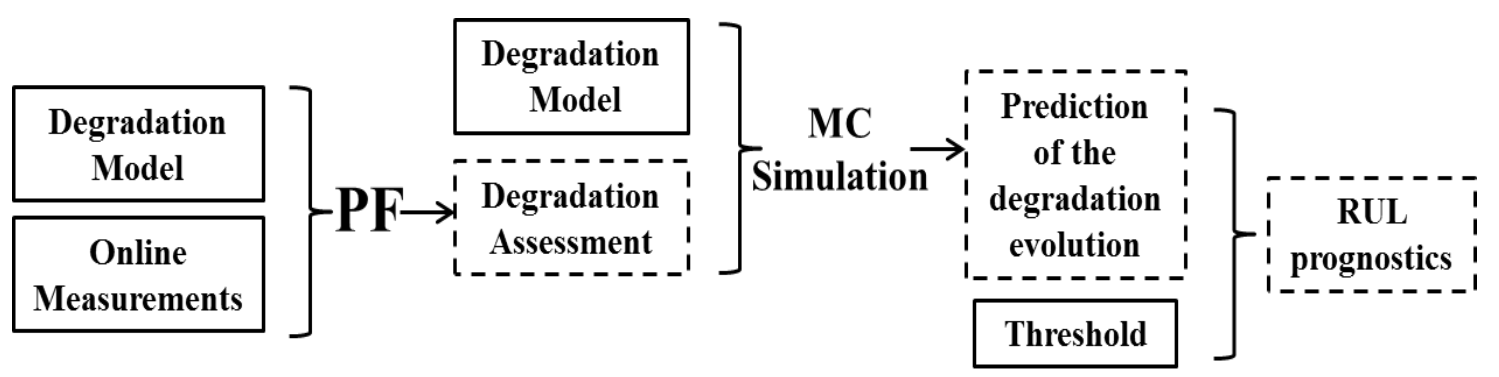

Figure 1. Sketch of the PF approach to fault prognostics

With respect to step 1), a natural framework for estimating the component degradation state and its RUL is offered by Bayesian filters ([6], [21], [23], [24] and [25]). They allow to properly treat the process and measurement uncertainties and to update the degradation state and RUL estimates each time a new degradation indicator measurement becomes available. The procedure is based on the repetition of a prediction and updating stage each time a measurement becomes available. In the prediction stage, $p\left(x_{t-1} \mid z_{1: t-1}\right)$ is known, and, by using Eq. (1), the prediction distribution of the degradation at the next time can be obtained from:

$$
p\left(x_{t} \mid z_{1: t-1}\right)=\int p\left(x_{t-1} \mid z_{1: t-1}\right) p\left(x_{t} \mid x_{t-1}\right) d x_{t-1}
$$


When the new measurement $z_{t}$ arrives, one can update and calculate the posterior pdf $p\left(x_{t} \mid z_{1: t}\right)$ using the Bayesian rule:

$$
p\left(x_{t} \mid z_{1: t}\right)=\frac{p\left(x_{t} \mid z_{1: t-1}\right) p\left(z_{t} \mid x_{t}\right)}{\int p\left(x_{t} \mid z_{1: t-1}\right) p\left(z_{t} \mid x_{t}\right) d x_{t}}
$$

Usually, except for the situation of linear Gaussian state space models (Kalman filter) and hidden finite-state space Markov chains (Wohnam filter), it is not possible to evaluate analytically the pdf in Eq. (4), since this requires the calculation of complex high-dimensional integrals. Particle Filter (PF) provides a numerical solution of the degradation state probability, which can be applied in the case of non-linear degradation models and nonGaussian non-additive noises. The PF solution is based on the Monte Carlo sampling of a large number of samples (called particles) from a proposal pdf $q\left(x_{t} \mid z_{1: t}\right)$. Then, the estimated posterior pdf $p\left(x_{t} \mid z_{1: t}\right)$ is approximated by:

$$
p\left(x_{t} \mid z_{1: t}\right) \approx \sum_{i=1}^{N} w_{t}^{i} \delta\left(x_{t}^{i}-x_{t}\right)
$$

where $x_{t}^{i}(i=1,2, \ldots, N)$ are the particles sampled from the proposal importance function distribution $q\left(x_{t} \mid z_{1: t}\right)$, $\delta$ is the Dirac delta function and $w_{t}^{i}$ is the weight associated to the particle $x_{t}^{i}$ given by:

$$
w_{t}^{\prime i}=\frac{p\left(z_{1: t} \mid x_{0: t}^{i}\right) p\left(x_{0: t}^{i}\right)}{q\left(x_{0: t}^{i} \mid z_{1: t}\right)}
$$

One of the most adopted choices is to consider the proposal importance function pdf $q\left(x_{t} \mid z_{1: t}\right)$ as the transition function, namely $q\left(x_{t} \mid z_{1: t}\right)=p\left(x_{t} \mid x_{t-1}\right)$. In this way, using Eq. (7), the particle weights $w_{t}^{i}$ at time $t$ are provided by: 


$$
w_{t}^{\prime i}=w_{t-1}^{\prime i} p\left(z_{t} \mid x_{t}^{i}\right) ; w_{t}^{i}=\frac{w_{t}^{i^{i}}}{\sum_{i=1}^{N} w_{t}^{\prime i}}
$$

where $p\left(z_{t} \mid x_{t}^{i}\right)$ is called the likelihood of measurement $z_{t}$ given the particle $x_{t}^{i}$, which can be derived from the observation function in Eq. (2). The reader interested in a detailed description of the PF method for the estimation of the degradation state can refer to [21], [26], [27], [28] and [29].

With respect to step 2), once the posterior pdf of the equipment degradation state has been estimated, it is possible to predict the future evolution of the equipment degradation trajectory by computing [30]:

$$
p\left(x_{t+l} \mid z_{1: t}\right)=\int \ldots \int \prod_{j=t+1}^{t+l} p\left(x_{j} \mid x_{j-1}\right) p\left(x_{t} \mid z_{1: t}\right) \prod_{j=t}^{t+l-1} d x_{j}
$$

where $p\left(x_{t+l} \mid z_{1: t}\right)$ is the predicted pdf of degradation state at time $t+l$. In order to facilitate this computation, according to [30], we numerically estimate the pdf of the degradation state at time $t+l, p\left(x_{t+l} \mid z_{1: t}\right)$ by:

$$
p\left(x_{t+l} \mid z_{1: t}\right) \approx \sum_{i=1}^{N} w_{t}^{i} \delta\left(x_{t+l}-x_{t+l}^{i}\right)
$$

where the particle state $x_{t+1}^{i}$ is obtained by iteratively applying Eq. (1) to the state $x_{t}^{i}$ at the previous time $t$.

Finally the estimate of the RUL pdf in step 3 is performed by [30], [31], [32], [33], [34] and [35]:

$$
p\left(\left.R U L\right|_{1: t}, x_{i}<x_{t h}\right) \approx \sum_{i=1}^{N} w_{t}^{i} \delta\left(R U L-R U L_{t}^{i}\right)
$$

where $R U L_{t}^{i}$ is the RUL associated to the $i$-th particle at the present time $t$ given by:

$$
R U L_{t}^{i}=\left\{\left(T_{t}^{i}-1-t\right) \mid g\left(x_{T_{t}^{i}-1}, \gamma\right)<x_{t h}, g\left(x_{T_{t}^{i}-1}, \gamma\right) \geq x_{t h}\right\}
$$


and the failure times $T_{t}^{i}$ can be found by iteratively applying Eq. (1) to simulate the particles evolution.

\section{Capacitor Degradation Model}

In this Section, we present the physics-based degradation model (Eq. 1) and the corresponding measurement equation (Eq. 2) for aluminum electrolytic capacitors working in variable operating conditions. The main degradation mechanism is caused by chemical reactions occurring inside the component, that induce the vaporization of the contained electrolyte. According to [11] and [15], ESR is a degradation indicator for capacitors operating in stationary operating conditions. In particular, from a physical point of view, the ESR can be considered as the sum of the inherent electrical resistances of the materials composing the capacitor.

According to [20], the ESR time evolution for a capacitor aging at constant temperature $T^{a g}$ is given by:

$$
\operatorname{ESR}_{t}\left(T^{a g}\right)=\operatorname{ESR}_{0}\left(T^{a g}\right) e^{C\left(T^{a g}\right) t}
$$

where $\operatorname{ESR}_{0}\left(T^{a g}\right)$ represents the initial ESR value of a capacitor at temperature $T^{a g}, t$ the age of the capacitor and $C\left(T^{a g}\right)$ a coefficient which defines the degradation rate of the capacitor. Resorting to the Arrhenius law, the temperature coefficient $C\left(T^{a g}\right)$ is given by:

$$
C\left(T^{a g}\right)=\frac{\ln 2}{\text { Life }_{\text {nom }} \cdot \exp \left[\frac{E_{a}}{k} \cdot \frac{T_{\text {nom }}-T^{a g}}{T_{n o m} \cdot T^{a g}}\right]}
$$

Where $E_{a}$ is the activation energy characteristic of the electrolyte, $k$ is the Boltzmann constant and Life $e_{\text {nom }}$ represents the nominal life of the capacitor aged at the constant nominal temperature $\left(T_{n o m}\right)$. A detailed description of the semi-empirical procedure adopted for the definition of the macro-level physical model of Eqs. (12) and (13) can be found in [19]. By applying Eq. (12), one can obtain the RUL of a capacitor operating at the constant temperature $T^{a g}$, for which, at the present time $t, \operatorname{ESR}_{t}\left(T^{a g}\right)$ is measured [20]: 


$$
R U L_{t}=t_{\text {fail }}-t=\frac{1}{C\left(T^{a g}\right)}\left[\ln \left(\frac{E S R_{t h}\left(T^{a g}\right)}{E S R_{t}\left(T^{a g}\right)}\right)\right]
$$

where $E S R_{t h}$ indicates the ESR value at which the capacitor is considered failed, usually considered as the double of its initial value $\operatorname{ESR}_{0}[18]$.

Notice, however, that Eq. (12) cannot be applied to a capacitor operating at variable temperatures since the measured ESR value depends on the temperature at which the measurement is performed, i.e., if we measure the ESR of the same capacitor at different temperatures, $T^{E S R}$, we obtain different ESR values. In the case of a new capacitor, the dependence of the ESR from the measurement temperature has been investigated by [20], who proposed the following model:

$$
\operatorname{ESR}_{0}\left(T^{E S R}\right)=\alpha+\beta e^{-\frac{T^{E S R}}{\gamma}}
$$

where $\alpha, \beta$ and $\gamma$ are parameters characteristics of the capacitor. Notice, however, that Eq. (15) does not apply to degraded capacitors and an analogous equation for degraded capacitors is not available. Thus, given the unavailability of a relationship between the measured ESR and the expected ESR at a reference temperature for a degraded capacitor, which would allow monitoring the degradation evolution, ESR "per se" is not a suitable degradation indicator for capacitors working at variable temperatures. For this reason, we take as a degradation indicator independent from the temperature at which ESR is measured, the ratio between the ESR measured at temperature $T^{E S R}$ and its expected initial value at the same temperature $T^{E S R}$ :

$$
\operatorname{ESR}_{t}^{\text {norm }}=\operatorname{ESR}_{t}\left(T^{E S R}\right) / \operatorname{ESR}_{0}\left(T^{E S R}\right)
$$

where $E S R_{0}\left(T^{E S R}\right)$ is obtained by using Eq. (15). Notice that the same degradation indicator, $E S R_{t}^{n o r m}$, would be associated to a degraded capacitor whose ESR value is measured at two different temperatures $T_{1}$ and $T_{2}$. In practice, the proposed degradation indicator allows overcoming the lack of knowledge on the relationship 
between the temperature and the measured ESR for a degraded capacitor, by considering the relative variation of the ESR with respect to that of a new capacitor at the same temperature.

Hence, the degradation process can be represented as a first order Markov Process between discrete time steps $t$ and $t-1$ :

$$
E S R_{t}^{n o r m}=E S R_{t-1}^{n o r m} e^{C\left(T_{t-1}^{a g}\right)}+\omega_{t-1}
$$

where $T_{t-1}^{a g}$ represents the aging temperature at time $t-1$ and $\omega_{t-1}$ models the process noise.

Notice that Eq. (17), which represents the degradation model (Eq. 1) in a sequential Bayesian approach, is independent from the measurement temperature $T^{E S R}$, but it depends from the temperature $T_{t-1}^{a g}$ experienced by the capacitor during its operation between time $t-1$ and $t$. Since the ESR measurement is performed during vehicle startup when the capacitor is in thermic equilibrium with the external temperature, whereas the capacitor aging occurs during motor operation when the capacitor temperature is higher, we will indicate the two capacitor temperatures with the two different symbols $T^{E S R}$ and $T^{a g}$.

The equation linking the measurement of the degradation indicator, $z_{t}=\operatorname{ESR}_{t}\left(T_{t}^{E S R}\right)$, and the degradation indicator, $E S R_{t}^{\text {norm }}$, is:

$$
z_{t}=\operatorname{ESR}_{t}^{n o r m} \cdot\left(\alpha+\beta e^{-\frac{\left(T_{t}^{E S R}-273.15\right)}{\gamma}}\right)+\eta_{t}
$$

where $T_{t}^{E S R}$ represents the measurement temperature at time $t$ and $\eta_{t}$ represents the measurement noise.

Notice that both temperatures, $T^{a g}$ and $T^{E S R}$, are quantities affected by a measurement error $\varepsilon_{T}$ :

$$
\begin{aligned}
& T_{\text {meas }}^{a g}=T_{\text {real }}^{a g}+\varepsilon_{T} \\
& T_{\text {meas }}^{E S R}=T_{\text {real }}^{E S R}+\varepsilon_{T}
\end{aligned}
$$


Under the non-additivity and non-gaussianity of the noise terms, $\varepsilon_{T}$, in Eqs. (17) and (18), a particle filter-based approach is applied for the estimate of the component degradation state at the present time. Then, the prediction of the future evolution of the degradation state is performed by Monte Carlo simulation, iteratively applying Eq. 17, where the noise on the aging temperature is properly sampled from the underlying distributions.

\section{CASE STUDIES}

In this Section, the proposed prognostic approach has been verified considering numerical simulations of the degradation process (Section 4.1) and experimental data collected during an accelerated degradation test (Section 4.2).

In both cases, we consider a capacitor of the ALS30 series in pristine conditions produced by KEMET. In order to properly set parameters $\alpha, \beta$ and $\gamma$ in Eq. (15) for this type of capacitor, experimental laboratory tests have been performed. In particular, ESR has been measured at different temperatures on a new capacitor using a FLUKE PM6306 RLC meter in a Votsch Industrietechnik climatic chamber. The experimental test procedure has been based on the following three steps:

- $\quad$ setting of the desired temperature in the climatic chamber;

- once stationary conditions are reached in the climatic chamber, the temperature is maintained for 20 minutes in order to allow the internal layers of the capacitor to heat up and reach the thermodynamic equilibrium with the chamber;

- the ESR is measured at different frequencies, between $10 \mathrm{kHz}$ and $1 \mathrm{MHz}$.

This procedure has been repeated at 7 different temperatures in the range [ $285 \mathrm{~K}, 383 \mathrm{~K}$ ], which is expected to be experienced by the capacitor during operation in a FEV. The measurement frequency of $20 \mathrm{kHz}$ has been selected given that the obtained results show that it is associated to the lowest ratio between noise and signal values. The results of the experimental tests are described in [36].

Parameters $\alpha, \beta$ and $\gamma$ have been set to the values reported in Table 1 by using the exponential regression method ([37] and [38]). 
Table 1. Experimental values for $\alpha, \beta$ and $\gamma$ parameters.

\begin{tabular}{|c|c|}
\hline$\alpha$ & $0.0817 \Omega$ \\
\hline$\beta$ & $0.037 \Omega$ \\
\hline$\gamma$ & $30.682 \mathrm{~K}$ \\
\hline
\end{tabular}

\subsection{Tests with simulated data}

The proposed prognostic method has been firstly applied to simulated data. The simulation of realistic capacitor degradation paths has required to simulate the operating conditions, i.e. the temperature profiles experienced by the FEV capacitor (Section 4.1.1), the degradation process (Section 4.1.2) and the measurement temperatures which influence the measured ESR values (Section 4.1.3).

\subsubsection{Simulation of the temperature profile}

Since real data describing the temperature experienced by a capacitor in a FEV are currently not available, possible temperature profiles have been simulated by taking into account the suggestions of experts of the motor behavior. The simulations are based on the following assumptions:

- $\quad$ the FEV is operating 3000 hours in a year (250 hours each month);

- the temperature experienced by the FEV capacitor has been simulated by adding $70 \mathrm{~K}$ to the external temperature in order to reproduce the effect of motor operation. For each day of the year, the external temperature has been sampled from a Gaussian distribution with mean and standard deviation equal to the historical mean and standard deviation of the month in Milan, Italy.

Figure 2 shows an example of simulated temperature evolution during a capacitor life. 


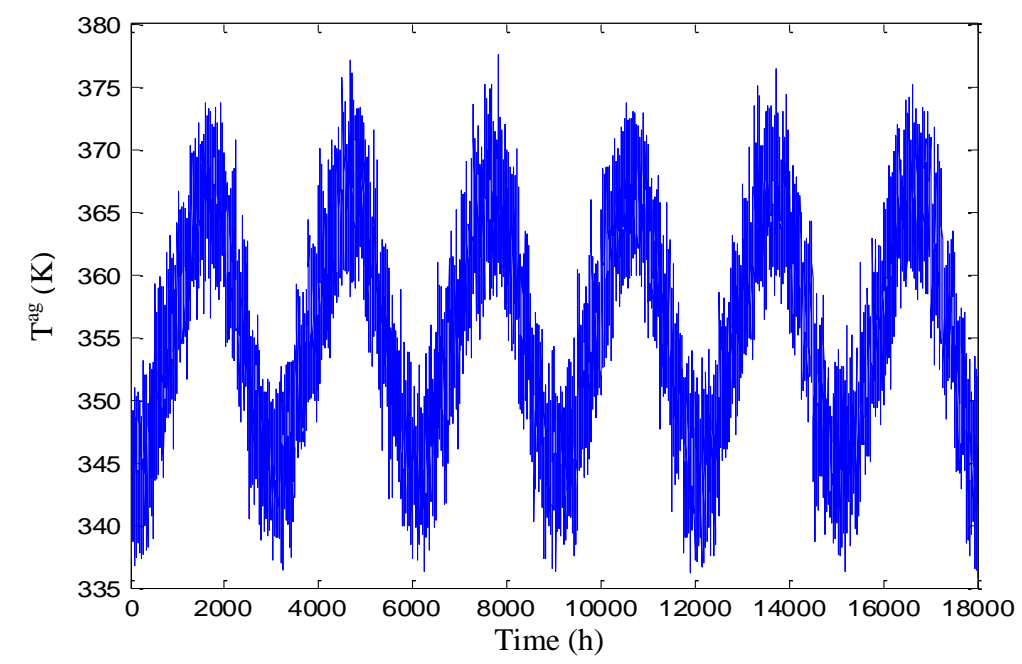

Figure 2. Numerical simulation of the temperature, $T^{a g}$, experienced by the capacitor during its life

\subsubsection{Simulation of the capacitor degradation process}

The numerical simulation of a possible capacitor degradation trajectory has been performed assuming an initial $E S R^{\text {norm }}$ value equal to $100 \%$ and iteratively applying Eq. (17) with a time step equal to 1 hour. The temperature values, $T^{a g}$, are those presented in Section 4.1.1; the process noise, $\omega_{t}$, describing the intrinsic uncertainty of the physical degradation process, has been sampled from a normal distribution with zero mean and standard deviation equal to $0.2 \%$. For the computation of the coefficient $C\left(T^{a g}\right)$, we have considered that the ALS30 Series electrolytic capacitor has a nominal life, Life $e_{n o m}$, of 20000 hours at the nominal aging temperature, $T_{n o m}^{a g}$, of $358 \mathrm{~K}$ [39]. The failure time of the capacitor is defined at the time at which $E S R_{n o r m}$ of the capacitor reaches the failure threshold of $200 \%$ [18]. Figure 3 shows a simulated evolution of the $E S R_{n o r m}$ value. 


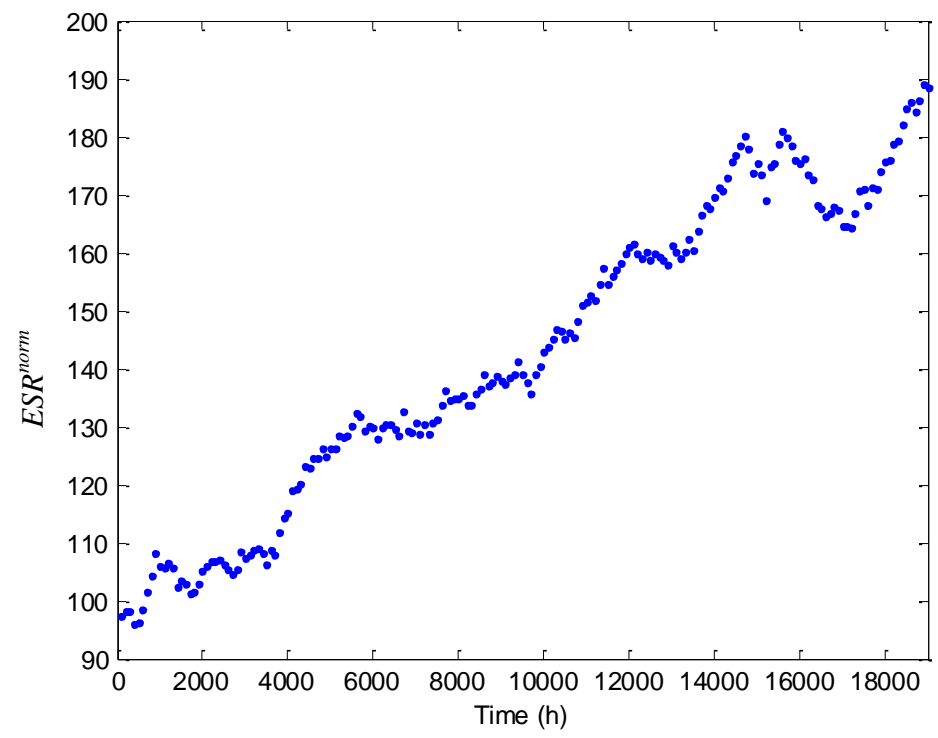

Figure 3. Numerical simulation of the $E S R^{\text {norm }}$ evolution

\subsubsection{Simulation of the ESR measurementmeasures}

With respect to the measurements, the measured ESR values, $z_{t}=E S R_{t}\left(T^{E S R}\right)$, have been obtained by applying Eq. (18) to the numerically simulated degradation indicator values $E S R^{\text {norm }}$ and considering the measurement noise, $\eta_{t}$, as a normal random variable with zero mean and standard deviation equal to $0.002 \Omega$.

Since in real FEV applications, the ESR will be measured only at the start-up of the vehicle, the capacitor temperature at the measurement time depends only on the external temperature. Thus, the measurement temperature $T^{E S R}$ has been simulated by adding to the external temperature profile described in Section 4.1.1 (equal to $T^{a g}-70 \mathrm{~K}$ ), a random value, $\varepsilon_{T}$, sampled from a normal distribution with zero mean and standard deviation equal to $3 \mathrm{~K}$, in order to reproduce the measurement error and the daily temperature variability. In this work $T^{E S R}$ and ESR measurements are assumed to be measured every 100 hours, starting from $t=100 \mathrm{~h}$ to $t=19000$ $\mathrm{h}$, whereas $T^{a g}$ is assumed to be measured every hour. 
Figure 4 shows the simulated values of the 190 ESR (left) and temperature (right) measurements corresponding to the degradation trajectory of Figure 3. Notice the significant difference between the evolution behavior of the proposed degradation indicator $\operatorname{ESR}^{\text {norm }}$ (Figure 3) and the corresponding ESR value (Figure 4, left).
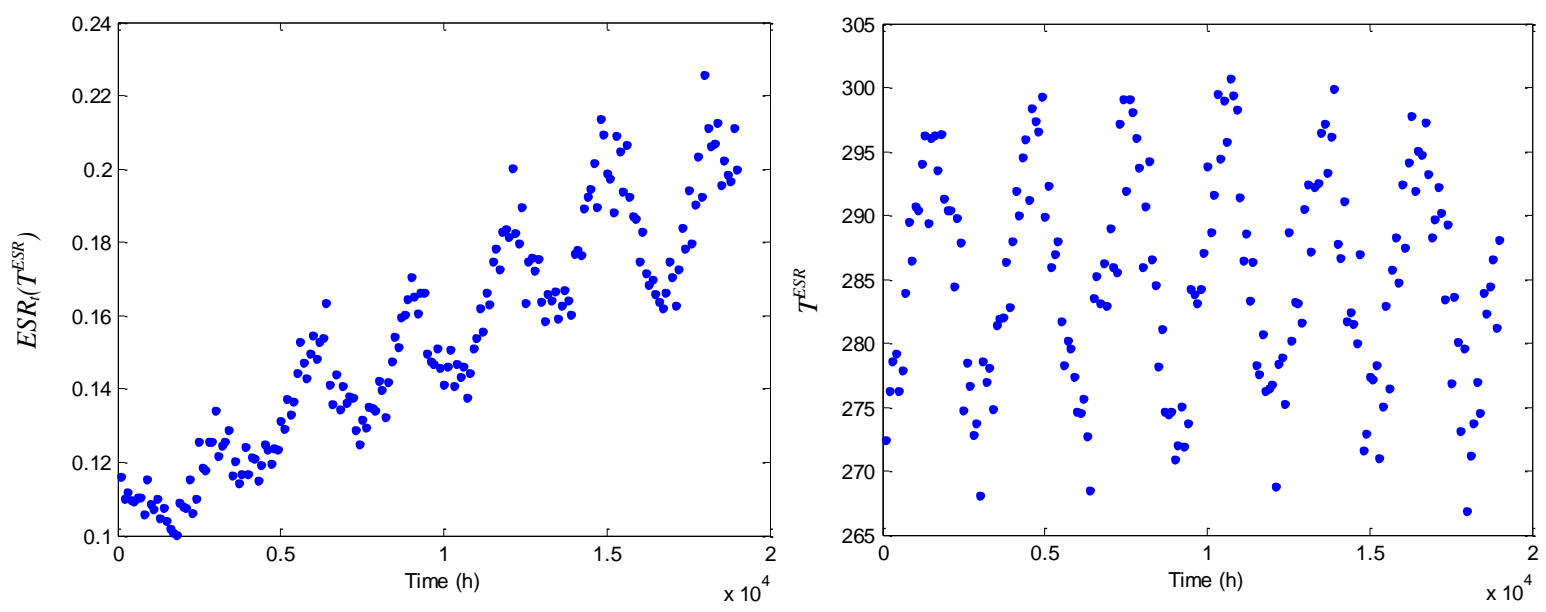

Figure 4. Numerical simulations of the measured ESR, $E S R_{t}\left(T^{E S R}\right)$ (left) and of the ESR measurement temperature, $T^{E S R}$ (right)

\subsubsection{Results}

The prognostic method described in Section 3 has been applied to the simulated capacitor life of Section 4.1.3 described by the 190 ESR and temperature measurements of Figure 4. In order to obtain a good compromise between accuracy and computational time, 1000 particles have been used for the PF simulation. The prognostic method provides a prediction of the RUL in the form of a probability density function (pdf) whose $10^{\text {th }}$ and $90^{\text {th }}$ percentiles, and the expected values are shown in Figure 5.

Notice that, as expected, the range of variability of the predicted RUL is decreasing with time: the $80 \%$ prediction interval size, between the estimated $10^{\text {th }}$ and $90^{\text {th }}$ percentiles, is progressively reducing from the first measurement $(t=100 \mathrm{~h})$ to the last measurement $(t=19000 \mathrm{~h})$. This reduction of the RUL uncertainty is due to: i) the acquired knowledge of the degradation provided by the ESR measurements, which allows updating the degradation probability distribution; ii) the reduction of the time horizon span, which makes the RUL prediction task less affected by the process uncertainty. 


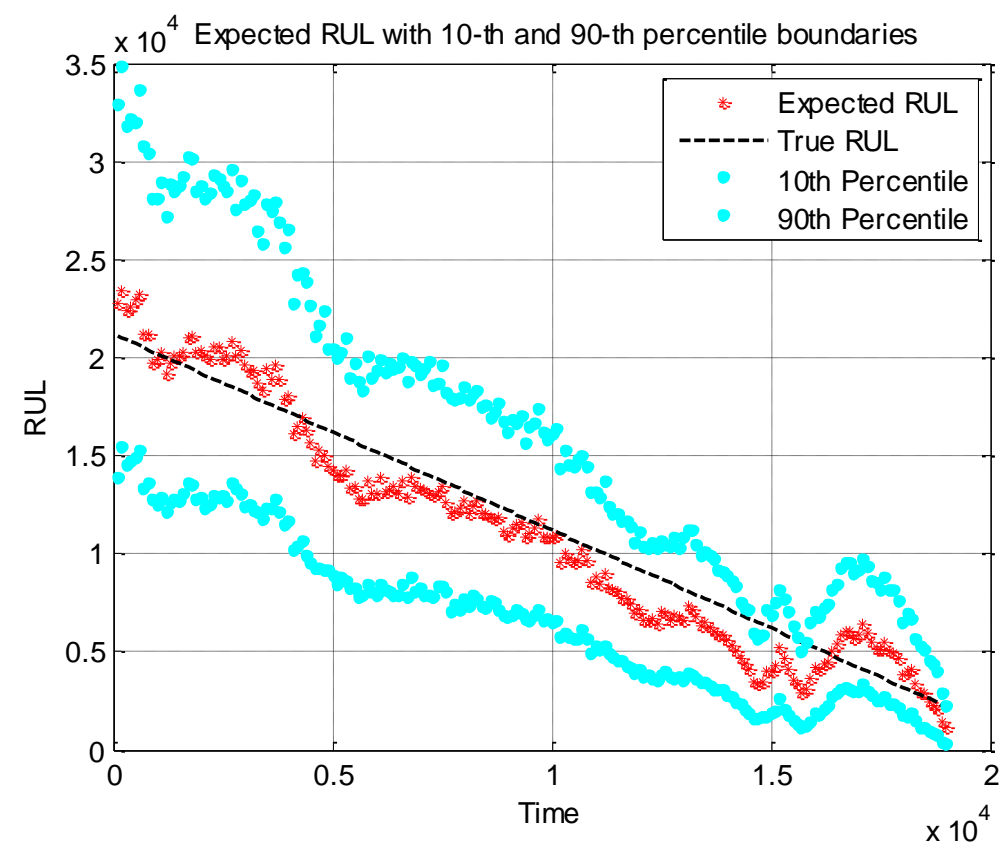

Figure 5. RUL prediction and corresponding 10 and $90 \%$ percentiles

The prognostic method has also been applied to other 100 capacitor degradation trajectories, all simulated by applying the procedure illustrated in Sections 4.1.1 - 4.1.3. The performance of the PF-algorithm has been verified with respect to five metrics: precision, accuracy, steadiness, coverage and risk level ([40] and [41]).

The Precision Index (PI) computes the relative width of the prediction interval, which is defined by:

$$
P I_{t}=\frac{\sup \left(R U L_{-} I_{t}\right)-\inf \left(R U L_{-} I_{t}\right)}{R U L_{t}}
$$

where $\sup \left(R U L_{-} I_{t}\right)$ and $\inf \left(R U L_{-} I_{t}\right)$ are the upper and lower bounds of the $80 \%$ RUL prediction interval and $R U L_{t}$ is the true RUL at time $t$. Small values of $P I_{t}$ indicate more precise predictions.

The Accuracy Index (AI) is defined as the relative error of the RUL prediction: 


$$
A I_{t}=\frac{\left|\overline{R U L_{t}}-R U L_{t}\right|}{R U L_{t}}
$$

where $\overline{R U L}$ is the RUL expected value at time $t$. Small values of $A I_{t}$ indicate more accurate predictions.

The Steadiness Index (SI) measures the volatility of the expected value of the failure time prediction $\bar{T}$ when new measurements become available. It is defined by:

$$
S I_{t}=\sqrt{\operatorname{var}\left(\bar{T}_{(t-\Delta t): t}\right)}
$$

where $\Delta t$ is the length of a sliding time window: in this paper, we take $\Delta t=100$. Small values of $S_{t}$ indicate more stable predictions.

The Risk Index (RI) is the probability of obtaining a RUL estimate smaller than the true RUL:

$$
R I_{t}=P\left(\overline{R U L} \leq R U L_{t}\right)=\int_{-\infty}^{R U L_{4}} p_{f}(R U L) d R U L
$$

where $p_{f}(R U L)$ is the estimate of the RUL pdf. Large $R I_{t}$ values indicate conservative RUL predictions, which are associated to lower risk from maintenance decisions.

The Coverage Index (COV) is a binary index which considers whether the true RUL lies within the $80 \%$ RUL prediction interval:

$$
C O V_{t}=\left(\inf \left(R U L_{-} I_{t}\right) \leq R U L_{t} \leq \sup \left(R U L_{-} I_{t}\right)\right)
$$

where $\sup \left(R U L_{-} I_{t}\right)$ and $\inf \left(R U L_{-} I_{t}\right)$ are the upper and lower bounds of the $80 \%$ RUL prediction interval and $R U L_{t}$ is the real RUL at time $t$. The average value of $C O V_{t}$ over the component life provides information on the ability of the prognostic method to represent the uncertainty on the prediction. Coverage values close to 0.8 indicate a good representation of the uncertainty [42]. 
Table 2 reports the average of the five performance metrics over 100 test trajectories. One can observe an average accuracy index around $35 \%$ and a high volatility of the predictions. Notice that the average accuracy on the estimation of the degradation state, $E S R^{\text {norm }}$, equal to 0.0085 is more satisfactory, indicating that the inaccuracy is mainly due to the stochasticity in the future evolution of the degradation trajectory, which renders the degradation process intrinsically difficult to predict. Furthermore, since the coverage is close to $80 \%$, one can observe that the true RUL tends to be within the $80 \%$ prediction interval. From these observations, we can conclude that the method is able to properly represent the uncertainty on the degradation process and can be used for maintenance decisions, albeit a relative error around $35 \%$.

Table 2. Average value of the Performance Indexes AI, PI, SI, RI, COV over 100 "real" trajectories.

\begin{tabular}{|c|c|c|}
\hline & RUL Prediction & $\boldsymbol{E S R}^{\text {norm }}$ Estimation \\
\hline Precision Index & 1.10 & 0.028 \\
\hline Accuracy Index & 0.35 & 0.0085 \\
\hline Steadiness Index & 303.02 & 0.51 \\
\hline Risk Index & 0.47 & \\
\hline Coverage Index & 0.82 & \\
\hline
\end{tabular}

Since in real applications the standard deviation of the process noise affecting the degradation process is typically unknown and in some cases it cannot be estimated considering the few real data available, we have investigated the performance of the PF method considering different values of the noise standard deviation. In particular, we have performed two additional runs of the PF algorithm, assuming $\sigma\left(\omega_{t}\right)=0.1$, and $\sigma\left(\omega_{t}\right)=0.3$, respectively. Table 3 lists the average of the five performance metrics over 100 test trajectories. 
Table 3. Average value of the Performance Indexes AI, PI, SI, RI, COV over 100 "real" trajectories.

\begin{tabular}{|c|c|c|c|}
\hline \multirow{2}{*}{} & \multicolumn{3}{|c|}{ PF Process Noise Standard Deviation } \\
\cline { 2 - 4 } & $\sigma\left(\omega_{\mathfrak{t}}\right)=\mathbf{0 . 1}$ & $\sigma\left(\omega_{\mathfrak{t}}\right)=\mathbf{0 . 2}$ & $\sigma\left(\omega_{\mathfrak{t}}\right)=\mathbf{0 . 3}$ \\
\hline Precision Index & 0.56 & 1.10 & 1.63 \\
\hline Accuracy Index & 0.34 & 0.35 & 0.37 \\
\hline Steadiness Index & 187.96 & 303.02 & 402.35 \\
\hline Risk Index & 0.43 & 0.47 & 0.51 \\
\hline Coverage Index & 0.48 & 0.82 & 0.96 \\
\hline
\end{tabular}

The effect of using a non-optimal setting of the process noise in the PF method is mainly on the precision and volatility of the prediction, and on the coverage of the prediction interval, whereas the other metrics such as the accuracy and the risk index are not significantly influenced. As expected, the higher the process noise used by the PF-based prognostic method, the larger the prediction interval and the higher the coverage. Thus, when we have to set the process noise in a PF-based prognostic method, we have to carefully evaluate the trade-off between using a small process noise variance, that results in small prediction intervals but too low coverage values, and a large process noise variance, that results in satisfactory coverage values but too large prediction intervals.

In practice, in those cases in which enough experimental data for estimating the process noise are not available, we can adopt a trial-and-error procedure. One can develop different PF-prognostic methods, characterized by different process noise standard deviation values and evaluate the coverage of the degradation state prediction on the available experimental data. A process noise standard deviation leading to a coverage close to the desired $80 \%$ should be preferred. 


\subsection{TESTS WITH EXPERIMENTAL DATA}

The accelerated degradation process has been performed at CEIT facilities in San Sebastian, Spain. The ALS30 series capacitor has been degraded in a Votsch Industrietechnik climatic chamber kept at the constant temperature of $418 \mathrm{~K}\left(T^{a g}\right)$. During this accelerated degradation test, the capacitor has been periodically taken out of the climatic chamber, cooled at room temperature $\left(298 \mathrm{~K}, T^{E S R}\right)$ and the ESR measured at the frequency of $20 \mathrm{kHz}$. Given the slowness of the degradation process and the time constraints on the duration of the tests, it has been possible to collect only 7 measurements of the capacitor ESR, with the last available measurement being equal to $130 \%$ of the initial ESR value. Thus, in order to evaluate the prediction performance of the developed algorithm, we have set the ESR failure threshold equal to $E S R^{\text {norm }}=130 \%$. The obtained ESR measurements are shown in Figure 6.

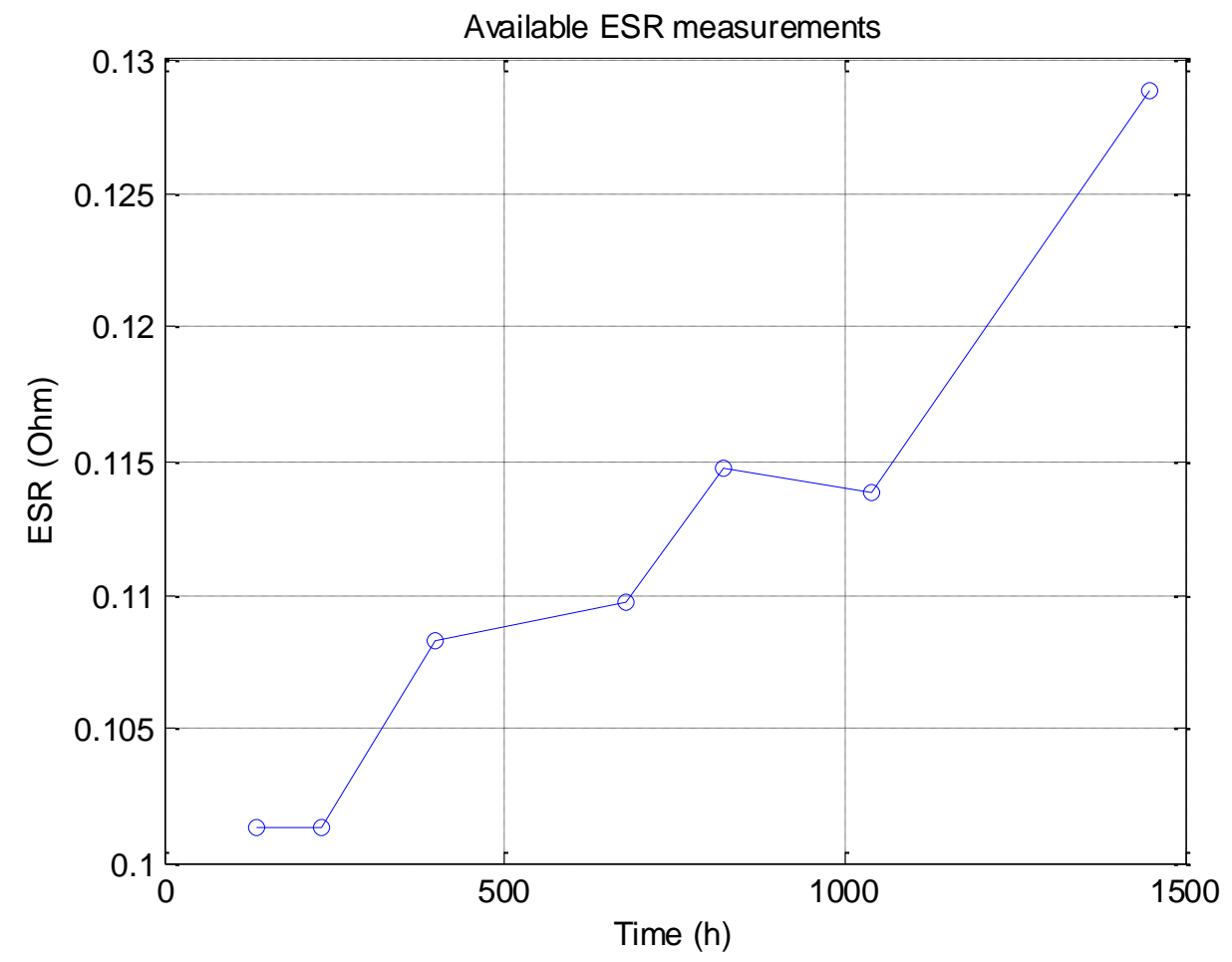

Figure 6 Measured value $\operatorname{ESR}_{t}\left(T^{E S R}\right)$ 


\subsubsection{Results}

We have applied the PF-based prognostic method described in Section 3 to these real degradation data considering 1000 particles and three different settings of the process noise standard deviation: 0.1, 0.2 and 0.3. The same parameters of the measurement error distributions used to test the numerical simulations in Section 4.1 have been used.

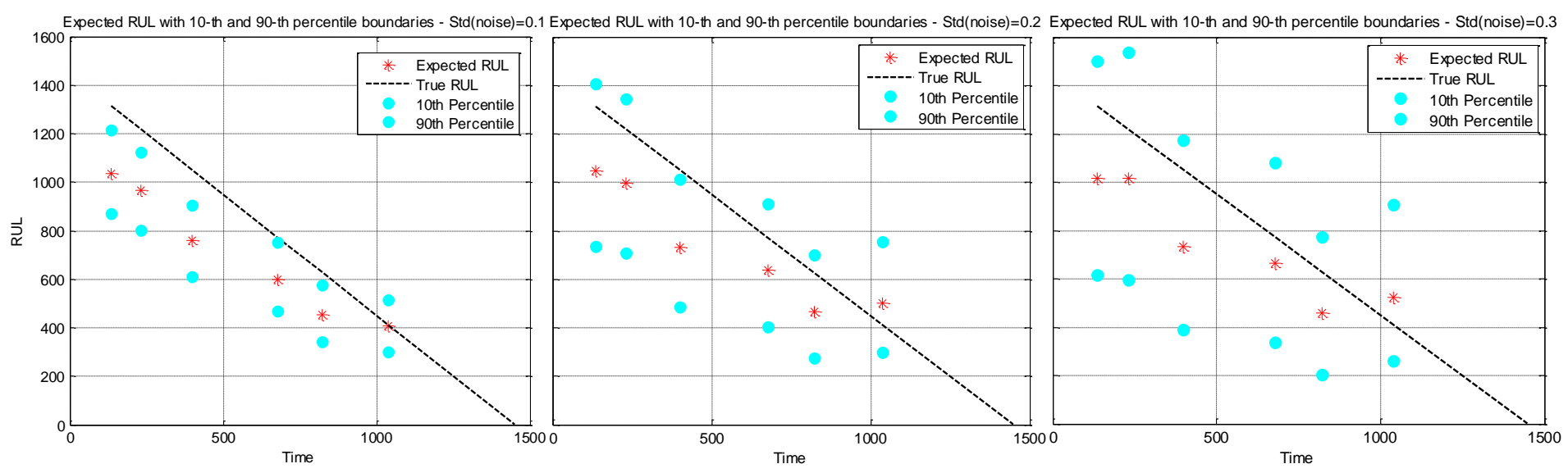

Figure 7. RUL prediction and corresponding $10^{\text {th }}$ and $90^{\text {th }}$ percentiles. The left Figure refers to a process noise standard deviation of 0.1 , the Figure in the middle to 0.2 , the right Figure to 0.3

Figure 7 shows the obtained RUL predictions and the corresponding $10^{\text {th }}$ and $90^{\text {th }}$ percentiles. Notice that in all the three cases, the RUL expected value tends to become closer to the true RUL value as new ESR measurements becomes available. It is also interesting to notice that, due to the decrease of the ESR measurement between time $822 \mathrm{~h}$ and time $1038 \mathrm{~h}$, the RUL predicted at time $1038 \mathrm{~h}$ results larger than that predicted at time $822 \mathrm{~h}$, in the cases in which process noise standard deviations of 0.2 and 0.3 are used, whereas a process noise standard deviation equal to 0.1 is less influenced by the lower ESR measurement.

Table 4 reports the five metrics previously considered to evaluate the prognostic performance of the method. The best performance is obtained by considering a process noise standard deviation equal to 0.2 , whereas using a standard deviation process noise equal to 0.1 we obtain a too low coverage value that indicates that the method is 
not properly taking into account the uncertainty, since the true RUL values turn out to fall outside the prediction intervals in many cases. On the other side, when a process noise standard deviation equal to 0.3 is considered, the uncertainty of the prediction is overestimated, leading to a coverage of $100 \%$ but with very large prediction intervals (the precision index is $42 \%$ larger than that obtained using a process noise standard deviation equal to 0.2). It is also interesting to observe that the process noise standard deviation does not have significant effects on the accuracy of the prognosis (Table 4, second line), but only on the representation of the prediction uncertainty.

Table 4. Average value of the Performance Indexes AI, PI, SI, RI, COV over the 6 available real ESR measurements

\begin{tabular}{|c|c|c|c|}
\hline \multirow{2}{*}{} & \multicolumn{3}{|c|}{ PF Process Noise Standard Deviation } \\
\cline { 2 - 4 } & $\sigma\left(\omega_{\mathrm{t}}\right)=\mathbf{0 . 1}$ & $\sigma\left(\omega_{\mathrm{t}}\right)=\mathbf{0 . 2}$ & $\sigma\left(\omega_{\mathrm{t}}\right)=\mathbf{0 . 3}$ \\
\hline Precision Index & 0.34 & 0.66 & 0.94 \\
\hline Accuracy Index & 0.20 & 0.22 & 0.23 \\
\hline Steadiness Index & 44.67 & 76.99 & 100.27 \\
\hline Risk Index & 0.89 & 0.76 & 0.71 \\
\hline Coverage Index & 0.17 & 0.83 & 1 \\
\hline
\end{tabular}

\section{Conclusion}

In this paper, we have addressed the problem of predicting the RUL for components working in variable operating conditions. For exemplification, we have considered the case of aluminum electrolytic capacitors used in FEVs. Given the non-stationary operating conditions and, particularly, the varying operational temperature experienced by this kind of component, we have proposed a new degradation indicator independent from temperature. The indicator is defined as the ratio between the ESR measured at temperature $T^{E S R}$ and its initial value at the same temperature $T^{E S R}$. Using a physics-based model of the degradation evolution, we have developed a Particle Filterbased modeling framework to predict the capacitor RUL and applied it to both simulated and real degradation 
data. We have, also, investigated the effects of the uncertainty of the degradation model associated to the process noise, performing a sensitivity analysis on few noise values and evaluating the corresponding performance by means of commonly used prognostic metrics. The satisfactory performance of the method on both simulated and real data encourages further developments towards industrial application. In particular, further laboratory experiments are being performed at CEIT facilities within the European Project HEMIS (www.hemis-eu.org), in order to collect data describing the capacitor degradation process in environmental conditions similar to those of a FEV.

\section{ACKNOWLEDGEMENT}

The research leading to these results has received funding from the European Community's Framework Programme (FP7/2007-2013) under grant agreement $n^{\circ}$ 314609. The authors are grateful for the support and contributions from other members of the HEMIS project consortium, from CEIT (Spain), IDIADA (Spain), Jema (Spain), MIRA (UK), Politecnico di Milano (Italy), VTT (Finland), and York EMC Services (UK). Further information can be found on the project website (www.hemis-eu.org).

The participation of Enrico Zio to this research is partially supported by the China NSFC under grant number 71231001. The participation of Piero Baraldi to this research is partially supported by the European Union Project INNovation through Human Factors in risk analysis and management (INNHF, $\underline{\text { www.innhf.eu}}$ ) funded by the $7^{\text {th }}$ framework program FP7-PEOPLE-2011- Initial Training Network: Marie-Curie Action. . The participation of Daniel Astigarraga to this research is partially supported by the Basque Government under grant number PRE_2014_2_200. 


\section{REFERENCE}

[1] J. Lee, J. Ni, D. Djurdjanovic, H. Qiu, and H. Liao, "Intelligent prognostics tools and e-maintenance", Computers in Industry, 57 (6), pp. 476-489, 2006.

[2] M.G. Pecht, Prognostics and Health Management of Electronics. Prognostics and Health Management of Electronics, 2008, pp. 1-315.

[3] E. Zio, "Prognostics and health management of industrial equipment", Diagnostics and Prognostics of Engineering Systems: Methods and Techniques, pp. 333-56, 2012.

[4] P. Baraldi, M. Compare, A. Despujols, and E. Zio, "Modelling the effects of maintenance on the degradation of a water-feeding turbo-pump of a nuclear power plant", Proceedings of the Institution of Mechanical Engineers, Part O: Journal of Risk and Reliability, 225 (2) , pp. 169-183, 2011.

[5] B. Ji, V. Pickert, W. Cao, and B. Zahawi, "In situ diagnostics and prognostics of wire bonding faults in IGBT modules for electric vehicle drives”, IEEE Transactions on Power Electronics, 28 (12), pp. 5568-5577, 2013.

[6] A. Doucet, "On sequential Monte Carlo methods for Bayesian filtering”, Dept. Eng., University of Cambridge, UK, Technical Report, 1998.

[7] A. Shrivastava, M.H. Azarian, C. Morillo, B. Sood, and M. Pecht, "Detection and reliability risks of counterfeit electrolytic capacitors”, IEEE Transactions on Reliability, 63 (2), pp. 468-479, 2014.

[8] J.R. Celaya, C.S. Kulkarni, G. Biswas, and K. Goebel, "Towards a model-based prognostics methodology for electrolytic capacitors: A case study based on electrical overstress accelerated aging”, International Journal of Prognostics and Health Management, 3 (2), 2012.

[9] E. Wolfgang, "Examples for failures in power electronics systems", ECPE Tutorial on Reliability of Power Electronic Systems, 2007.

[10] K. Harada, A. Katsuki, and M. Fujiwara, "Use of ESR for deterioration diagnosis of electrolytic capacitors", IEEE Transactions on Power Electronics, vol. 8, no. 4, pp. 355-361, 1993.

[11] M. L. Gasperi, "Life prediction model for aluminum electrolytic capacitors", in Conference Record - IAS Annual Meeting (IEEE Industry Applications Society), 1996, 3, pp. 1347-1351. 
[12] B. Alvsten, "Electrolytic capacitors theory and application”, RIFA Electrolytic Capacitors, Sweden, 1995.

[13] C. Kulkarni, G. Biswas, X. Koutsoukos, J. Celaya, and K. Goebel, "Integrated diagnostic/prognostic experimental setup for capacitor degradation and health monitoring”, IEEE AUTOTESTCON, 2010.

[14] A. Lahyani, P. Venet, G. Grellet, and P.J. Viverge, "Failure prediction of electrolytic capacitors during operation of a switch-mode power supply", IEEE Transactions on Power Electronics, 13 (6), pp. 1199-1207, 1998.

[15] M. Hao and L. Wang, "Fault diagnosis and failure prediction of aluminum electrolytic capacitors in power electronic converters", Industrial Electronics Society, IECON 2005, 31st Annual Conference of IEEE, 2005, pp. 6.

[16] A. Braham, A. Lahyani, P. Venet, and N. Rejeb, "Recent developments in fault detection and power loss estimation of electrolytic capacitors”, IEEE Transactions on Power Electronics, 25 (1), pp. 33-43, 2010.

[17] M.A. Vogelsberger, T. Wiesinger, and H. Ertl, "Life-cycle monitoring and voltage-managing unit for DClink electrolytic capacitors in PWM converters”, IEEE Transactions on Power Electronics, 26 (2), pp. 493$503,2011$.

[18] P. Venet, H. Darnand, and G. Grellet, "Detection of faults of filter capacitors in a converter. Application to predictive maintenance", in Proc. Int. Telecommun. Energy Conf., 1993, 74, pp. 229-234.

[19] F. Perisse, P. Venet, G. Rojat, and J.M. Rétif, "Simple model of an electrolytic capacitor taking into account the temperature and aging time", Electrical Engineering, 88 (2), pp. 89-95, 2006.

[20] K. Abdennadher, P. Venet, G. Rojat, J.M. Rétif, and C. Rosset, “A real-time predictive-maintenance system of aluminum electrolytic capacitors used in uninterrupted power supplies", IEEE Transactions on Industry Applications, 46 (4), pp. 1644-1652, 2010.

[21] M.S. Arulampalam, S. Maskell, N. Gordon, and T. Clapp, “A tutorial on particle filters for online nonlinear/non-Gaussian Bayesian tracking”, IEEE Transactions on Signal Processing, 50 (2), pp. 174-188, 2002.

[22] Y. Hu, , P. Baraldi, , F. Di Maio, and E. Zio, “A particle filtering and kernel smoothing-based approach for new design component prognostics”, Reliability Engineering \& System Safety, 2014. 
[23] B.D. Anderson and J.B. Moore, Optimal filtering. Englewood Cliffs (NJ): Prentice Hall, 1979.

[24] A. Doucet, J.F.G. De Freitas, and N.J. Gordon, Sequential Monte Carlo methods in practice. New York: Springer-Verlag, 2001.

[25] P. Lall, R. Lowe, and K. Goebel, "Extended kalman filter models and resistance spectroscopy for prognostication and health monitoring of lead free electronics under vibration", IEEE Transactions on Reliability, 61 (4), pp. 858-871, 2012.

[26] A. Doucet, N. J. Gordon, and V. Krishnamurthy, "Particle filters for state estimation of jump Markov linear systems", IEEE Transactions on Signal Processing, 49(3), pp. 613-624, 2001.

[27] W. He, N. Williard, M. Osterman, and M. Pecht, "Prognostics of lithium-ion batteries based on DempsterShafer theory and the Bayesian Monte Carlo method", Journal of Power Sources, 196 (23), pp. 10314$10321,2011$.

[28] F. Gustafsson, "Particle filter theory and practice with positioning applications", IEEE Aerospace and Electronic Systems Magazine, 25 (7 Part 2), pp. 53-81, 2010.

[29] F. Cadini, E. Zio, and D. Avram, "Monte Carlo-based filtering for fatigue crack growth estimation", Probabilistic Engineering Mechanics, 24 (3), pp. 367-373, 2009.

[30] A. Doucet, S. Godsill, and C. Andrieu, "On sequential Monte Carlo sampling methods for Bayesian filtering”, Statistics and Computing, 10 (3), pp. 197-208, 2000.

[31] M.E. Orchard and G.J. Vachtsevanos, "A particle-filtering approach for on-line fault diagnosis and failure prognosis", Transactions of the Institute of Measurement and Control, 31 (3-4), pp. 221-246, 2009.

[32] B. Saha, K. Goebel, S. Poll, and J. Christophersen, "Prognostics methods for battery health monitoring using a Bayesian framework", IEEE Transactions on Instrumentation and Measurement, 58 (2), pp. 291-296, 2009.

[33] P. Baraldi, F. Cadini, F. Mangili, and E. Zio, "Model-based and data-driven prognostics under different available information", Probabilistic Engineering Mechanics, 32, pp. 66-79, 2013.

[34] E. Zio and G. Peloni, "Particle filtering prognostic estimation of the remaining useful life of nonlinear components”, Reliability Engineering \& System Safety, 96, pp. 403-9, 2011. 
[35] D. An, J.H. Choi, and N.H Kim, "Prognostics 101: A tutorial for particle filter-based prognostics algorithm using Matlab”, Reliability Engineering and System Safety, 115, pp. 161-169, 2013.

[36] M. Rigamonti, P. Baraldi, E. Zio, D. Astigarraga, and A. Galarza, “A Particle Filtering-Based Approach for the Prediction of the Remaining Useful Life of an Aluminum Electrolytic Capacitor", in PHME 2014 Conference Proceedings, Volume 5, July 8th-10th, Nantes, France, 2014.

[37] A.C. Cameron and F.A.G. Windmeijer, "An R-squared measure of goodness of fit for some common nonlinear regression models", Journal of Econometrics, 77 (2), pp. 329-342, 1997.

[38] F. Di Maio, K.L. Tsui, and E. Zio, “Combining Relevance Vector Machines and exponential regression for bearing residual life estimation”, Mechanical Systems and Signal Processing, 31, pp. 405-427, 2012.

[39] KEMET Electronics Corporation, "Screw Terminal Aluminum Electrolytic Capacitors ALS30/31 Series", Technical Notes, pp. 1-14, 2014

[40] M. Orchard, F. Tobar, and G. Vachtsevanos, "Outer feedback correction loops in particle filtering-based prognostic algorithms, Statistical performance comparison”, Studies in Informatics and Control, 18, pp. 295304, 2009.

[41] B.E. Olivares, C. Munoz, M.E. Orchard, and J.F.Silva, "Particle-filtering-based prognosis framework for energy storage devices with a statistical characterization of state-of-health regeneration phenomena", IEEE Transactions on Instrumentation and Measurement, 62, pp. 364-76, 2013.

[42] P. Baraldi, F. Mangili, and E. Zio, "Investigation of uncertainty treatment capability of model-based and data-driven prognostic methods using simulated data", Reliability Engineering \& System Safety, 112, pp. 94108, 2013. 